\title{
Comparison of Chicken Cecal Microbiota after Metaphylactic Treatment or Following Administration of Feed Additives in a Broiler Farm with Enterococcal Spondylitis History
}

\author{
Julia Hankel 1,*(1), Björn Bodmann ${ }^{2}$, Matthias Todte ${ }^{2}$, Eric Galvez ${ }^{3,4}$, Till Strowig ${ }^{3,4}{ }^{\mathbb{D}}$, Dimitri Radko ${ }^{5}$, \\ Ali Antakli ${ }^{5}$ and Christian Visscher ${ }^{1}$ (D) \\ 1 Institute for Animal Nutrition, University of Veterinary Medicine Hannover, Foundation, Hannover, \\ Bischofsholer Damm 15, D-30173 Hannover, Germany; christian.visscher@tiho-hannover.de \\ 2 Tierarztpraxis MMT, Leopoldstraße 116, D-06366 Köthen, Germany; bjoern.bodmann@tap-mmt.de (B.B.); \\ matthias.todte@tap-mmt.de (M.T.) \\ 3 Helmholtz Center for Infection Research, Inhoffenstraße 7, D-38124 Braunschweig, Germany; \\ Eric.Galvez@helmholtz-hzi.de (E.G.); till.strowig@helmholtz-hzi.de (T.S.) \\ 4 Hannover Medical School, Carl-Neuberg-Straße 1, D-30625 Hannover, Germany \\ 5 Elanco Animal Health GmbH, Werner-Reimers-Straße 2-3, D-61352 Bad Homburg, Germany; \\ radko_dmytro@elanco.com (D.R.); ali_antakli@elanco.com (A.A.) \\ * Correspondence: julia.hankel@tiho-hannover.de
}

Citation: Hankel, J.; Bodmann, B.;

Todte, M.; Galvez, E.; Strowig, T.;

Radko, D.; Antakli, A.; Visscher, C.

Comparison of Chicken Cecal

Microbiota after Metaphylactic

Treatment or Following

Administration of Feed Additives in a

Broiler Farm with Enterococcal

Spondylitis History. Pathogens 2021,

10, 1068. https://doi.org/10.3390/

pathogens 10081068

Academic Editor:

Antonio José Piantino Ferreira

Received: 24 June 2021

Accepted: 19 August 2021

Published: 23 August 2021

Publisher's Note: MDPI stays neutral with regard to jurisdictional claims in published maps and institutional affiliations.

Copyright: () 2021 by the authors. Licensee MDPI, Basel, Switzerland. This article is an open access article distributed under the terms and conditions of the Creative Commons Attribution (CC BY) license (https:// creativecommons.org/licenses/by/ $4.0 /)$.

\begin{abstract}
Minimizing the clinical signs of Enterococcus cecorum infections causing enterococcal spondylitis in broiler herds is successful when initiated as metaphylaxis in the first week of life. Mechanistically, either the Enterococcus species present at that time are reduced by antibiotic treatment or antibiotic treatment might induce changes in intestinal microbiota composition with an indirect and subsequent influence. The aim of the present study was to examine the cecal microbiota of chickens after administering lincospectin or different additives to evaluate whether these additives have lincospectin-like effects on microbiota. Therefore, 157,400 broiler chickens were reared in four chicken houses ( 40,000 birds each) on a broiler farm with history of enterococcal spondylitis. Each flock was treated either with lincospectin or water soluble esterified butyrins, Bacillus (B.) licheniformis or palm oil was added via drinking water during the first days of life. Ten birds per house were dissected at days 11, 20 and 33 of life and cecal microbiota were analyzed (16S rRNA gene sequencing). Lincospectin treatment elicited significant changes in the cecal microbiota composition until slaughter age. Among the tested additives, effects of $B$. licheniformis on cecal microbiota composition were most similar to those seen after the treatment with lincospectin at day 11.
\end{abstract}

Keywords: $16 \mathrm{~S}$ rRNA gene; beta diversity; bacterial pathogens; poultry

\section{Introduction}

Enterococcus (E.) cecorum infections with a clinical course have been increasingly reported in different countries worldwide [1-3], forming an important emerging disease in modern broiler chickens associated with arthritis and osteomyelitis and leading to high mortality rates $[1,2,4]$. Lesions of osteomyelitis of the caudal thoracic vertebrae compressing the spinal cord and/or arthritis of the hock, stifle and coxofemoral joints provoked clinical signs, and E. cecorum was always isolated from these lesions [2,3]. The disease has been called enterococcal spondylitis, based on the frequent isolation of E. cecorum from the lesions and necrosis and inflammation observed in the free thoracic vertebrae of affected birds [5]. E. cecorum with pathogenic genotypes were identified in the intestines of naturally infected birds as early as week one, in contrast to commensal E. cecorum strains that did not appear until week three [6]. This ability to colonize the gut early in life may provide pathogenic E. cecorum strains with a competitive advantage and potentiate dissemination throughout a flock [6]. 
The onset of clinical signs like paresis and lameness [2,3] usually occurs at the beginning of week three of life $[4,6]$, resulting in up to $7 \%$ increased mortality $[2,3]$. Disease outbreaks caused by enterococci are considered opportunistic [7] but the exact origin, predisposing factors and pathogenesis of enterococcal-associated vertebral osteoarthritis are still largely unknown [7-9]. It is suggested that intestinal colonization, bacteremia and osteochondrosis dissecans of the free thoracic vertebra in early life are crucial for the pathogenesis of enterococcal spondylitis [6]. Common features in epidemiology and clinical presentation have led several authors to suggest mechanisms by which this gastrointestinal inhabitant enters the bloodstream. Disturbance of the normal gut microbial balance could increase numbers of E. cecorum in the chicken intestine, and thus its prevalence in the poultry house environment and invasion of the systemic circulation might occur subsequent to damage to the respiratory, intestinal or integumentary barriers [8]. It is suggested that entry of E. cecorum into the bloodstream may occur because of gastrointestinal stress [5] or following disruption of the gut mucosal barrier caused by a previous enteric disease, such as coccidiosis or bacterial enteritis [6,8], as well as mechanical or toxic irritants [6]. Recently, it could be shown that Enterococcus spp. might enhance the survival of other pathogenic intestinal bacteria and co-infections, leading to increased virulence [10]. In contrast, infections with other intestinal pathogens decrease bacteremia and spinal lesions caused by pathogenic E. cecorum [11]. These observations indicate interactions of Enterococcus spp. with other intestinal bacteria. Furthermore, even if it is suggested that disruption of the intestinal structure could potentiate E. cecorum bacteremia, it was not observed that clinical intestinal diseases necessarily cause E. cecorum bacteremia [6].

Diseased flocks are often treated with one or more antibiotics. However, the effect of antibiotic therapy is temporary [7] or only successful when initiated as metaphylaxis from the first week of life [2]. Additionally, several Enterococcus species seem to be resistant against the most frequently used antibiotics in poultry $[12,13]$. Against this background, mechanisms affecting E. cecorum other than the direct bactericidal or bacteriostatic ones could be suspected. This led to the assumption that early antibiotic treatment might induce substantial changes to the microbiota composition of the intestines with the consequence of an indirect and subsequent influence on this bacterial species and the course of the disease. As E. cecorum-positive spleens were found as early as week 1 and during weeks 2 and 3 respectively [6], the suspected most likely time window of bacterial translocation from the gut became the focus of the present investigation of intestinal microbiota composition (represented by day 11).

Antibiotics need to be used more prudently and alternatives are needed in animal agriculture, while high-throughput technologies could help to better understand effects and mechanisms of action of the various components guiding the selection of antibiotic alternatives [14]. Some used alternatives to antibiotics in agricultural animals are feed additives, which include prebiotics, probiotics, and organic acids [14]. Studies have shown that butyric acid preparations might be useful adjuncts to reduce necrotic enteritis in antibiotic-free broiler production [15]. Their mechanism of action against the disease must involve other host-mediated activities as there was no direct microbial inhibition of Clostridium perfringens [16]. Butyric acid is an important energy source for gut epithelial cells and stimulates epithelial cell proliferation and differentiation [17-19], it has well documented anti-inflammatory effects [20], promotes concentration-dependent intestinal barrier function $[21,22]$ and demonstrates beneficial effects against intestinal bacteria with zoonotic relevance in poultry production $[23,24]$. Some commercially available probiotic Bacillus strains, including Bacillus (B.) licheniformis, were effective at inhibiting pathogenic E. cecorum in vitro [25] and the administration of B. licheniformis was able to normalize ileum microbiota disorders caused by necrotic enteritis in chickens [26]. Finally, plant oils containing medium-chain fatty acids showed in vitro antibacterial activity against gram-positive intestinal bacteria, such as E. cecorum while the same oils did not show any effect on commensal bacteria (Bifidobacterium spp. and Lactobacillus spp.) [27]. 
The aim of the present investigation was to examine microbiota changes due to the metaphylactic administration of lincospectin, and to ascertain whether using water soluble esterified butyrins, B. licheniformis or palm oil have lasting effects on the cecal microbiota of broiler chickens similar to those seen after treatment with lincospectin.

\section{Results}

\subsection{General Health and Performance Parameters}

The fattening period in chicken houses one, three and four ran without complications. At day 28 of life, chickens in chicken house two (esterified butyrins) were treated with colistin sulfate (Colistinsulfat 100, bela-pharm GmbH \& Co. KG, Vechta, Germany, $300 \mathrm{~g} / 1000 \mathrm{~L}$ ) for three days because of a systemic E. coli infection.

Average body weights measured at the slaughterhouse (total body weight of chickens divided by their number) met breeder performance objectives with the exception of chicken house 3 and 4 at days 28 and 33, which remained below expectations (Table 1).

Table 1. Average body weights of chickens (g) measured at slaughterhouse after depletion of chicken houses in batches and at the end of the fattening period.

\begin{tabular}{ccccc}
\hline Day of Life & Chicken House $\mathbf{1}$ & Chicken House $\mathbf{2}$ & Chicken House $\mathbf{3}$ & Chicken House $\mathbf{4}$ \\
\hline 28 & $1538(n=7285)$ & $1559(n=7113)$ & $1457(n=7025)$ & \\
33 & $1977(n=4958)$ & $1984(n=4954)$ & $1920(n=5006)$ & $1902(n=8255)$ \\
37 & $2350(n=25,025)$ & $2424(n=23,854)$ & & \\
38 & & & $2444(n=24,677)$ & $2467(n=28,224)$ \\
\hline
\end{tabular}

Breeder performance objectives: $1501 \mathrm{~g}$ at day $28,1956 \mathrm{~g}$ at day $33,2334 \mathrm{~g}$ at day 37 and $2429 \mathrm{~g}$ at day 38 [28].

\section{2. $16 S$ rRNA Gene Analyses}

After the filtering step, two further samples were removed from the dataset because of high dissimilarity to all other samples at day 33. Therefore, 115 of the obtained 120 samples were included in the statistical analyses of microbiota. The dataset contained 2,216,872 reads (mean number of reads: 19,277; range: 10,344-95,833) mapped to 541 operational taxonomic units (OTUs).

At day 11, the contribution of the factor treatment was highest, explaining $25.2 \%$ of the sample variability (Table 2). With increasing age, the influence of this factor on the microbial composition of the samples decreased.

Table 2. PERMANOVA testing the factor treatment on microbial composition of cecal samples taken at days 11,20 and 33 .

\begin{tabular}{ccccccc}
\hline & \multicolumn{2}{c}{ Day 11 } & \multicolumn{2}{c}{ Day 20 } & \multicolumn{2}{c}{ Day 33 } \\
\hline \multirow{3}{*}{ Treatment } & $\mathrm{R}^{2}$ & $\operatorname{Pr}(>\mathrm{F})$ & $\mathrm{R}^{2}$ & $\operatorname{Pr}(>\mathrm{F})$ & $\mathrm{R}^{2}$ & $\operatorname{Pr}(>\mathrm{F})$ \\
& 0.2520 & 0.001 & 0.2430 & 0.001 & 0.1667 & 0.001 \\
\hline
\end{tabular}

The microbiota composition of the cecal samples of the birds treated with lincospectin differed from the three additives. Bray-Curtis distances revealed that with increasing chicken age or time after treatment, dissimilarity in cecal microbiota composition between chickens treated with lincospectin and the other chickens decreased (Table 3, Figure 1). Chickens that had received $B$. licheniformis showed the highest similarity with lincospectintreated chickens at day 11, followed by chickens treated with esterified butyrins. 
Table 3. ANOSIM results (Statistic R values with a range from 0 (similar) to 1 (dissimilar)) on BrayCurtis distances between bacterial communities associated with treatments based on pairwise test.

\begin{tabular}{lcccc}
\hline & & B. licheniformis & Esterified Butyrins & Palm Oil \\
\hline \multirow{3}{*}{ Lincospectin } & Day 11 & $0.4110^{* * *}$ & $0.4778^{* * *}$ & $0.7518^{* * *}$ \\
& Day 20 & $0.7355^{* * *}$ & $0.6055^{* * *}$ & $0.5668^{* * *}$ \\
& Day 33 & $0.1854^{* * *}$ & $0.1770^{* * *}$ & $0.3005^{* * *}$ \\
\hline
\end{tabular}

*** Significance value: $p \leq 0.001$.

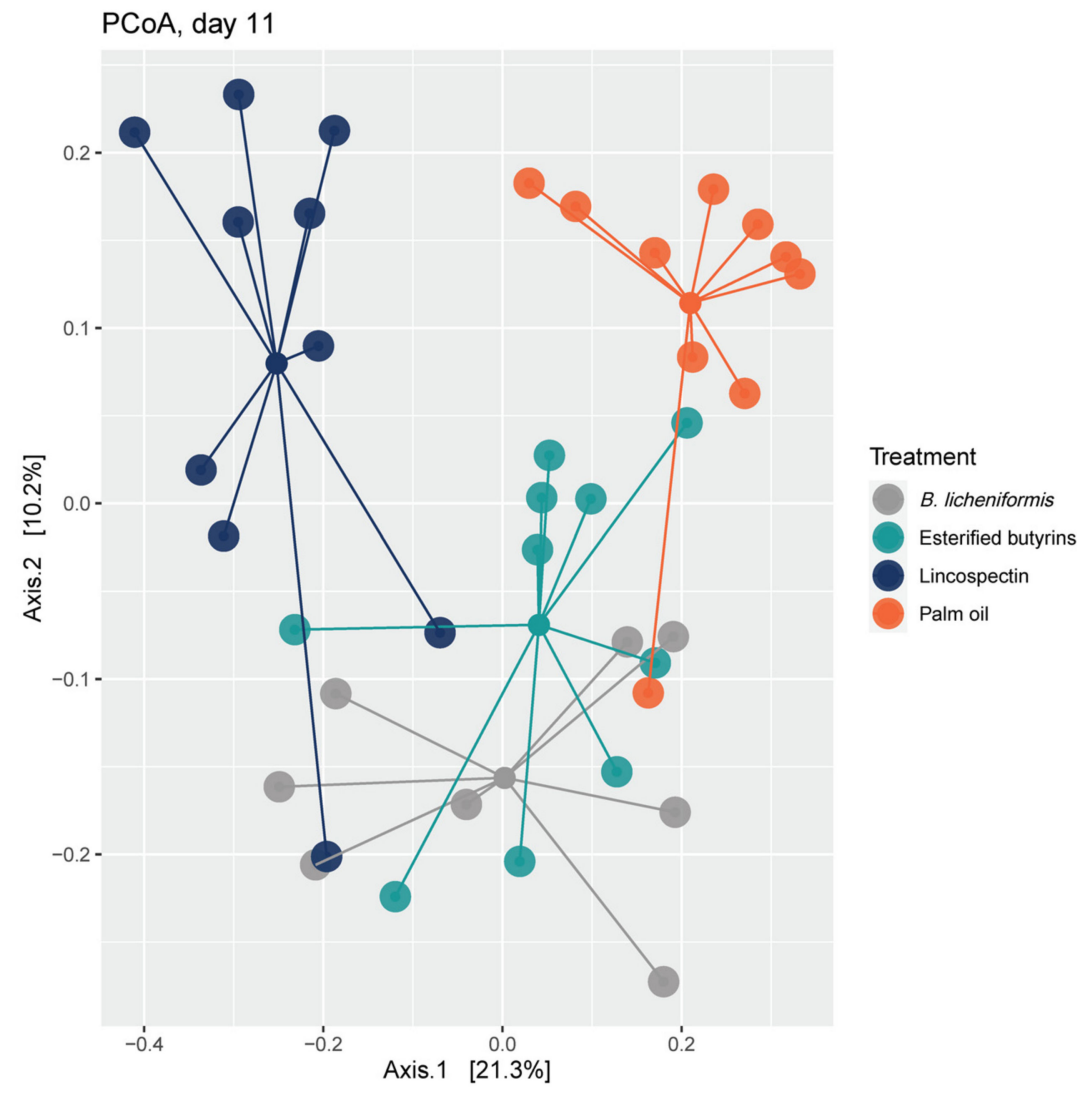

(a)
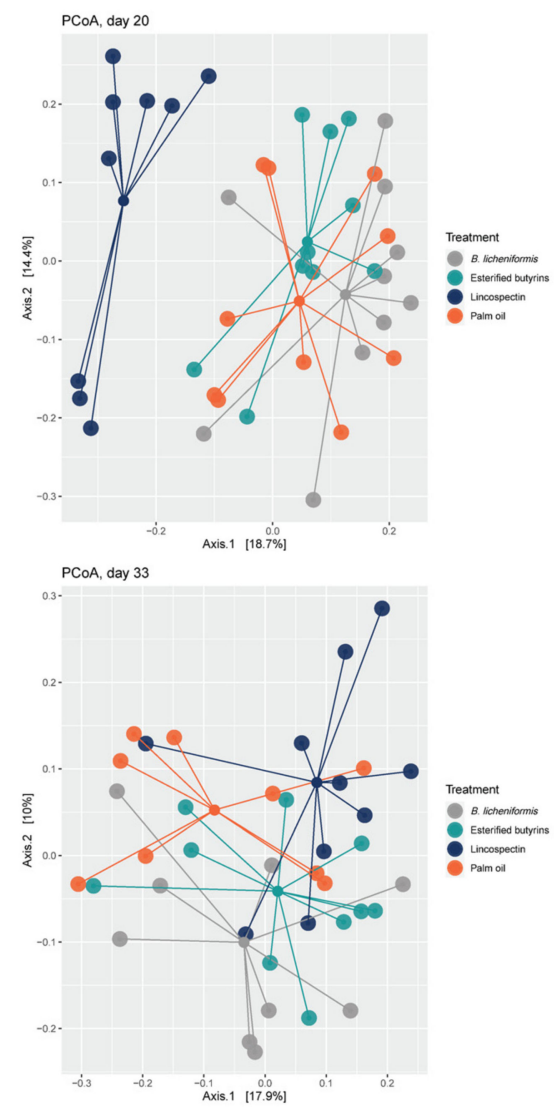

(b)

Figure 1. Bray-Curtis dissimilarity-based principal coordinate analysis (PCoA) was performed on cecal samples collected at: (a) day 11, (b) days 20 and 33. Each point represents a different bird; colored lines connect birds of one treatment (B. licheniformis, esterified butyrins, lincospectin and palm oil).

Comparison of the measured species richness estimators, observed species, Chao 1 and Shannon index in the cecal contents of the birds is shown in Figure 2. The treatment with lincospectin during the first days of the chicken's life lowered species richness significantly compared to the administered additives until day 33, while significant effects of lincospectin on species evenness was only seen at day 11 . At day 11, the Shannon index demonstrated that richness and evenness of cecal bacteria in chickens that had received B. licheniformis and esterified butyrins were highest. Nevertheless, species richness estimators revealed no statistically significant differences between the three additives at any of the measured time points (Table S1). 


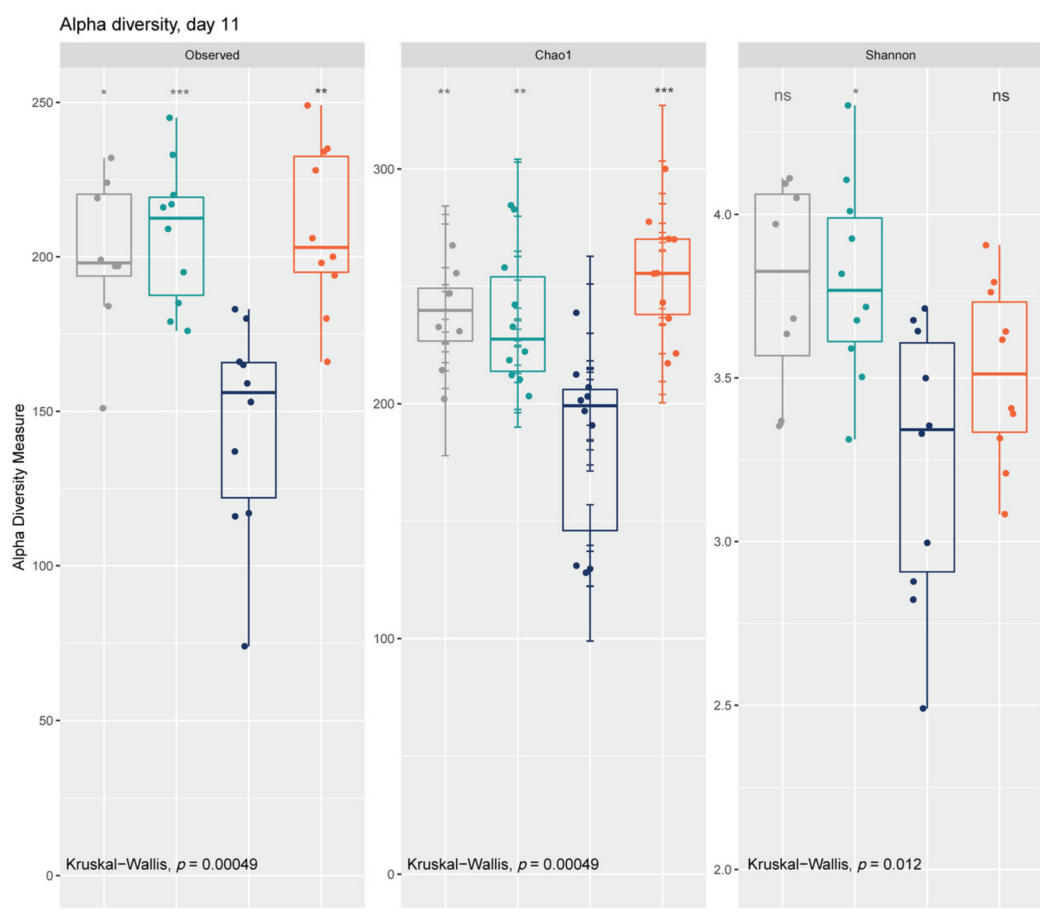

(a)

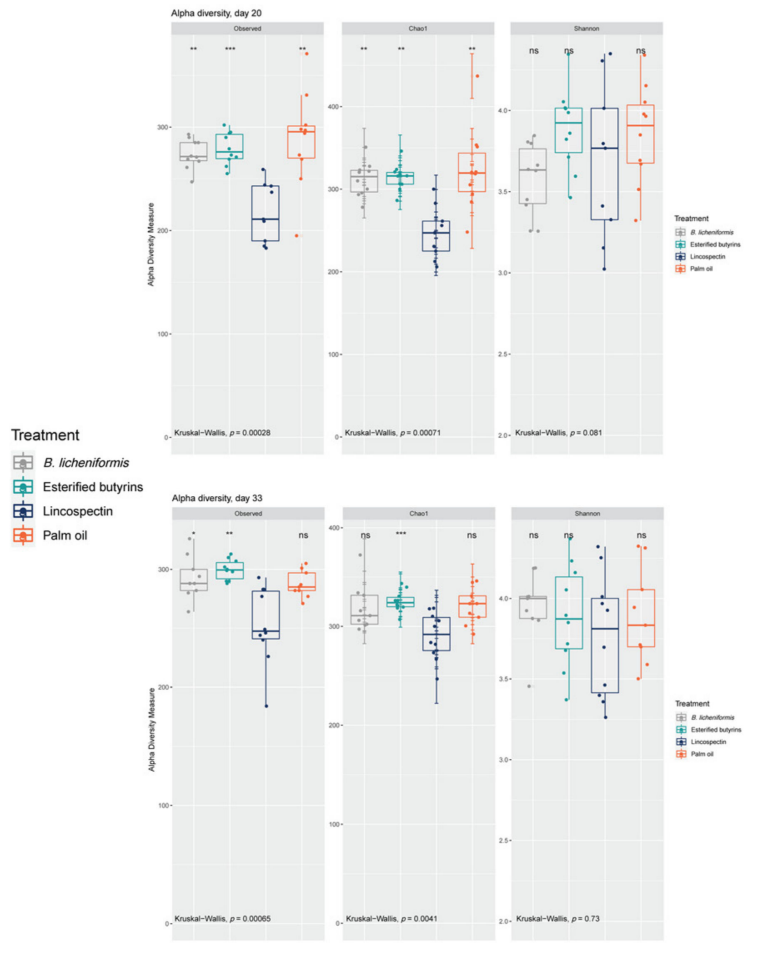

(b)

Figure 2. Boxplots showing alpha diversity in cecal samples using the species richness estimators observed species, Chao1 and Shannon index at: (a) day 11, (b) days 20 and 33 (ns: $p>0.05, *: p \leq 0.05,{ }^{* *}: p \leq 0.01,{ }^{* * *}: p \leq 0.001$ ).

At day 11, the microbiota of the cecal contents was dominated at the phylum level by Firmicutes, Tenericutes and Bacteroidetes. Relative abundance of Bacteroidetes was the highest and relative abundance of Firmicutes was the lowest in the cecal samples of the chickens that had received palm oil (Figure 3), resulting in the lowest Firmicutes to Bacteroidetes ratio. The cecal microbiota of chickens in the other groups were dominated by Firmicutes. The statistical analyses for the differences in bacterial family abundance revealed that chickens treated with lincospectin showed reduced abundance of the families Bacteroidaceae (OTU_2), uncultured rumen bacterium (OTU_57, Order: Mollicutes RF9), Defluviitaleaceae (OTU_277), Ambiguous_taxa (OTU_121, Order: Clostridiales), and Christensenellaceae (OTU_102) in comparison to the groups receiving one of the three feed additives (Table S2 and Figure S1). The greatest influence of lincospectin was seen on the family Bacteroidaceae showing an up to 4096-fold lower abundance compared to administering palm oil. After administering the feed additives several families belonging to the order Clostridiales (class: Clostridia, phylum: Firmicutes) were enriched compared to lincospectin treatment. In contrast, chickens that were treated with lincospectin showed an enriched abundance of the families Bifidobacteriaceae (OTU_76) and Staphylococcaceae (OTU_208) compared to all three administered feed additives. Bifidobacteriaceae abundance became 62-fold (palm oil), 276-fold (B. licheniformis), and 413-fold higher (esterified butyrins) due to the lincospectin treatment. 


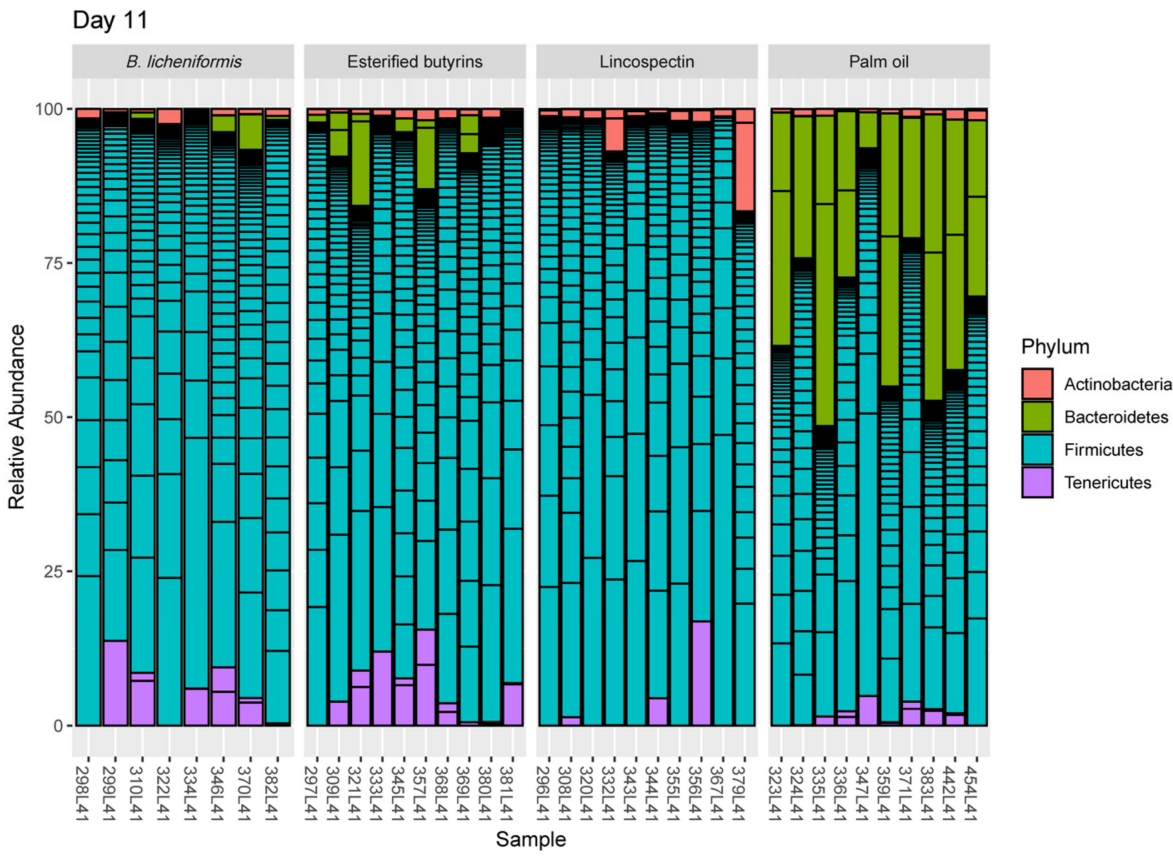

(a)

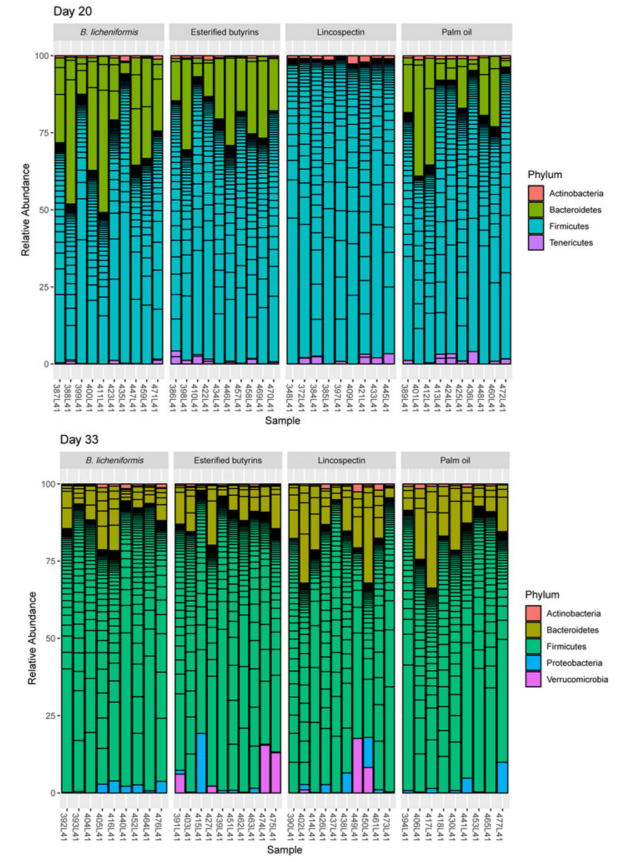

(b)

Figure 3. Bar charts represent the relative abundances of the dominant phyla comprising 50 of the most dominant OTUs in cecal samples of chickens at an age of (a) 11, (b) 20 and 33 days.

At day 20, relative abundance of Bacteroidetes in the cecal contents of the chickens that had received $B$. licheniformis and esterified butyrins increased, while the cecal microbiota of the lincospectin-treated chickens was still dominated by the phylum Firmicutes (Figure 3). At day 33, relative abundance of Firmicutes in cecal samples of chickens treated with lincospectin had decreased while Bacteroidetes increased. This development is reflected in a shift of the Firmicutes to Bacteroidetes ratio giving a similar picture between the groups at day 33 (Figure 3).

Members of the bacterial family Enterococcaceae were present from day 11 at the latest. With regard to the relative abundance of the family Enterococcaceae, no differences between the groups were found at day 11 (Table 4).

Table 4. Relative abundances (mean $\%, \pm \mathrm{sd}$ ) of selected bacterial families in cecal samples taken at day 11 .

\begin{tabular}{ccccc}
\hline Day 11 & \multicolumn{3}{c}{} \\
\hline Family & B. licheniformis & $\begin{array}{c}\text { Esterified } \\
\text { Butyrins }\end{array}$ & Lincospectin & Palm Oil \\
\hline Bacteroidaceae & $0.955 \pm 1.41$ & $3.15 \pm 3.96$ & $0.010 \pm 0.007$ & $24.7 \pm 11.1$ \\
Ruminococcaceae & $57.0 \pm 13.5$ & $57.5 \pm 6.10$ & $41.1 \pm 10.7$ & $45.9 \pm 9.60$ \\
Lachnospiraceae & $17.8 \pm 14.7$ & $14.2 \pm 4.77$ & $29.5 \pm 10.8$ & $12.5 \pm 4.22$ \\
Erysipelotrichaceae & $3.38 \pm 1.89$ & $4.64 \pm 3.60$ & $13.2 \pm 13.0$ & $3.29 \pm 1.77$ \\
Bifidobacteriaceae & $0.005 \pm 0.011$ & $0.005 \pm 0.009$ & $1.94 \pm 3.54$ & $0.026 \pm 0.063$ \\
Staphylococcaceae & 0 & $0.002 \pm 0.004$ & $0.179 \pm 0.209$ & $0.011 \pm 0.017$ \\
Enterococcaceae & $0.031 \pm 0.035$ & $0.158 \pm 0.157$ & $0.207 \pm 0.172$ & $0.110 \pm 0.231$ \\
\hline
\end{tabular}

\section{Discussion}

Due to the observed effect of the metaphylactic use of lincospectin by poultry veterinarians to prevent enterococcal spondylitis (personal communication), the present study aimed to first describe the intestinal microbiota composition under this treatment before comparing it with the intestinal microbiota of chickens after administering different feed additives. 


\subsection{Microbiota Composition under Lincospectin Treatment}

The administration of all treatments occurred only during the first days of the chicken's life, but the microbiota composition was influenced significantly until slaughter age. At day 11 , the factor treatment explained $25.2 \%$ of the variability in microbial composition and persisted until slaughter age (about 30 days after withdrawal of the treatments), where the factor treatment still explained $16.7 \%$ of the microbial composition variability. PERMANOVA revealed that antibiotic treatment (low-dose concentration) of turkey poults caused a change in community composition at day 6 , to a lesser extent than in the present study $\left(R^{2}=0.151, p=0.001\right)$ [29]. Antibiotic treatment with lincospectin and colistin enteromix of six-month old pigs induced significant differences in community structure as well (beta diversity of OTUs was assessed by PERMANOVA), whereby only $1.8 \%$ of sample's variability could be explained by this factor $\left(R^{2}=0.018, p=0.037\right)$ [30]. Age at the time of antibiotic administration appears to play a role in the extent to which administration affects intestinal microbiota composition. Single early-life antibiotic administration can alter the microbiota that persist long after exposure has ceased [31].

Richness in cecal samples of lincospectin-treated chickens was significantly affected, showing a lower richness compared to the other additives until slaughter age. This speciesreducing effect of antibiotic treatment is a widely reported [32-34] and therefore expected effect. Evenness of the present bacterial species was less affected with advanced age as indicated by the Shannon index. This indicates that the cecal contents of lincospectintreated chickens hosted less varied bacterial species but with similar even distribution compared to the other chickens that had received one of the three additives. The cecal microbiota of chickens treated with lincospectin had not only an overall lower richness but also a clear dominance of Firmicutes with low proportions of Bacteroidetes until at least day 20. Similar results have already been shown in humans where the level of functional diversity is linked to the relative abundance of Bacteroidetes, and microbiota enriched for Firmicutes / Actinobacteria have a lower level of functional diversity [35]. Diversity of the cecal microbiota in the present study increased generally with increasing age. This development was expected as the early cecal microbiota is characterized by poor diversity but gains complexity and matures to a stable and diverse microbiota with increasing age [36]. The number of genera found in the chicken ceca doubled from day 7 to day 42 [37]. The cecal microbiota of chickens are more susceptible to changes as they are developing during half of the production period [36]. For this reason, an accelerated development of chicken gut microbiota can be considered beneficial with regard to resistance to several intestinal pathogens or the outcome of infectious diseases. This could be shown already for Salmonella Enteritidis [38] and Campylobacter jejuni [23,39]. The treatment with lincospectin in the present study seemed to retard this microbiota development. With regard to potentially pathogenic Enterococcus (E.) cecorum strains, this effect of lincospectin on microbiota development should be less advantageous, which does not correspond to the effects seen in practice (personal communication with poultry veterinarians). Diseased flocks are often treated with one or more antibiotics. However, the effects of administering antibiotics seem to be only successful when initiated as a metaphylaxis from the first week of life onwards [2]. This supports the suspected importance of the early period of the life of broiler chickens in the pathogenesis of enterococcal spondylitis [6]. Therefore, it can be assumed that pathogenic E. cecorum strains appear to be distinct from the other chicken intestinal pathogens, which generally colonize broiler flocks later (in the case of Campylobacter jejuni with an age of 3-4 weeks $[40,41])$ or only interact with a certain bacterial community.

\subsection{Microbiota Maturation under Administering of Palm Oil}

The microbiota composition of chickens that had received palm oil was the one with the largest difference in the microbiota composition of chickens that had been treated with lincospectin. At day 11, the relative abundance of Firmicutes and Bacteroidetes in chickens that had received palm oil clearly differed from all other groups. The Bacteroidetes to Firmicutes ratio changes with age, as seen in the present study. Proteobacteria is the first most 
abundant phylum in the cecal contents of chickens (the remaining part of cecal microbiota is formed by representatives of the family Lachnospiraceae), while within the following days, the families Lachnospiraceae and Ruminococcaceae (phylum Firmicutes) gain predominance, which is in turn taken over by Bacteroidetes with advanced age, reaching a constant ratio of Bacteroidetes and Firmicutes formed by equal numbers of the representatives of both phyla in adult chickens [42-44]. Even if Firmicutes and Bacteroidetes are usually equally represented in the cecal microbiota of healthy adult chickens, there is still high individual variation and individual chickens with $10 \%$ to $90 \%$ Bacteroidetes in their microbiota exist without exhibiting any signs of abnormal behavior [42]. The major families from Firmicutes colonized chicken ceca are Lachnospiraceae and Ruminococcaceae, followed by Lactobacillaceae, Veillonellaceae and Erysipelotrichaceae [42]. The abundance of the families Lachnospiraceae and Erysipelotrichaceae were enriched in the lincospectin-treated chicks compared to chicks that had received palm oil $(p<0.05$, Table S2). All members of the family Lachnospiraceae are anaerobic, fermentative, and chemoorganotrophic, while some have strong hydrolyzing activities, e.g., through carbohydrate-active enzymes [45]. Therefore, Lachnospiraceae have a considerable capacity to utilize diet-derived polysaccharides (including starch, inulin, and arabinoxylan) and further degrade components of plant material (cellulose and hemicellulose) which are fermented and converted to short-chain fatty acids (SCFAs) like acetate, butyrate, and propionate $[46,47]$. The SCFAs can be absorbed and used for energy by the host [47]. Additionally, SCFAs and the biosynthesis of bacterial metabolites from aromatic amino acid metabolism by Lachnospiraceae strengthens the intestinal barrier and are associated with the maintenance of gut health $[46,47]$. Within the phylum Firmicutes, members of the families Lachnospiraceae and Ruminococcaceae have been shown to express enzymes favoring the production of butyrate over propionate, while representatives of the phylum Bacteroidetes are mainly propionate producers [43]. Several genus belonging to the family Erysipelotrichaceae are capable of fermentation of carbohydrates to butyrate [42]. Butyrate has direct trophic effects, improves epithelial integrity and defense systems and has also been implicated in the down-regulation of bacterial virulence, which is why butyrate is a helpful feed additive in animal production, especially when ingested soon after birth, as it controls gut health disorders caused by bacterial pathogens (reviewed in Guilloteau et al. [48]).

The aerobic atmosphere of hatcheries, farms and animal houses can contain spores of Clostridiales, families Lachnospiraceae and Ruminococcaceae, which is why both families colonize soon after E. coli in newly hatched chicks [42]. The likelihood of a chicken being colonized by Bifidobacteriaceae is low and therefore a longer time is needed for their appearance in gut microbiota [42]. About three days post hatch Bifidobacteriaceae rise is thought to be an important step in the maturation of cecal microbiota as it stimulates the growth of Bacteroidaceae [36]. Even if the treatment with lincospectin seemed to retard microbiota development in the present study, the abundance of Bifidobacteriaceae was significantly enriched in lincospectin-treated chickens compared to chickens that had received one of the other feed additives at day 11. Bifidobacteriaceae play an important role in pathogen exclusion and intestinal barrier function, with most of the evidence coming from mammalian studies [36], while in chickens the role of Bifidobacterium has not yet been fully elucidated and the benefit of cecum Bifidobacteriaceae colonization in chickens needs to be further clarified $[49,50]$. Bifidobacterium species are not numerically dominant in chickens [42,49], but are used as probiotics mainly in humans but also in chickens [51]. After in ovo supplementation of Bifidobacteria strains, serum amino transferases were not affected which led the authors to the assumption that this observation might be connected with the reduction effect of Bifidobacteria on the translocation of harmful bacteria in the gut and liver [52]. Since the invasion of pathogenic E. cecorum strains into the systemic circulation is thought to occur subsequent to the disruption of the gut mucosal barrier [6,8], altering intestinal microbiota composition during the first days of life in favor of bacterial families which promote intestinal barrier integrity should be beneficial and represent a possible mechanism of action of lincospectin against this disease. 
It can be supposed that adding palm oil to drinking water during the first days of the chicken's life promoted microbiota maturation compared to the other additives and the treatment with lincospectin, which might be a disadvantage with regard to potentially pathogen E. cecorum strains. Nevertheless, it should be emphasized that the possible effects of the additives under E. cecorum infection were not evaluated in the present study, no statement can be made in this respect.

\subsection{Similar Microbiota Composition in Chickens Administered B. Licheniformis, Esterified Butyrins and Lincospectin}

Among the tested additives, the effects of B. licheniformis on cecal microbiota composition were most similar to those seen after the treatment with lincospectin at day 11 . Administering the probiotic strain B. licheniformis did not result in differed cecal bacterial diversity compared to administering esterified butyrins or palm oil but induced a clear dominance of Firmicutes with low proportions of Bacteroidetes at day 11, this being very similar to the esterified butyrins and lincospectin-treated groups. Similarly, this observation of the abundance of the phylum Firmicutes and of the genus Lactobacillus in excreta increased in broilers fed B. licheniformis-fermented products, whereas the abundance of the phyla Verrucomicrobia and Bacteroidetes decreased in response to the treatment [53]. Trela et al. [54] were equally unsurprised about the diversity indices being lower after $B$. licheniformis supplementation because of the induced increase in the proliferation of Firmicutes. Administering B. licheniformis seems to induce a modulation of the $\mathrm{pH}$ value within the gastrointestinal tracts of chickens [54,55], which is suspected to be a cause of the induced alterations in microbiota composition by B. licheniformis [54]. In the present study, members of the bacterial family Enterococcaceae were present from day 11 at the latest. No differences in the relative abundance of Enterococcaceae were found between the groups at day 11 , whereby relative abundance in chickens that had received B. licheniformis were the lowest. Additionally, Chen, Ying-Chu and Yu [53] observed a decreased abundance of the genus Enterococcus in the excreta of broilers fed B. licheniformis-fermented products. Additionally, some commercially available probiotic Bacillus strains were effective at inhibiting pathogenic E. cecorum in vitro [25]. Considering these observations with the results of the present study, this feed additive is very interesting for studying E. cecorum infection with a standardized approach.

\section{Materials and Methods}

\subsection{Experimental Design, Animals and Housing, Sampling}

In total, 157,400 broiler chickens of the breed Ross 308 were supplied as day-old chicks from the same hatchery and reared on one fattening farm in Germany with enterococcal spondylitis history in four chicken houses ( 40,000 birds each) for about five weeks (37 to 38 days). One identical conventional complete diet was offered ad libitum in all chicken houses. Chickens in each chicken house were treated either with lincospectin or one of three different additives administered via drinking water during the first days of life. Chickens in the first chicken house were treated with lincospectin (Lincospectin ${ }^{\circledR}$ Pulver, Zoetis Deutschland GmbH, Berlin, Germany, $250 \mathrm{~g} / 1000 \mathrm{~L}$ ) at the first day of life up to the age of two days (three days in total). At the first day of life up to the age of three days (four days in total), water soluble esterified butyrins were administered to the chickens in the second chicken house $(0.12-0.24 \mathrm{~mL} / \mathrm{kg}$ body weight). Over the same period, the chickens in the third chicken house were offered drinking water containing Bacillus licheniformis $(70 \mathrm{~g} / 1000 \mathrm{~L})$, while palm oil $(2 \mathrm{~L} / 1000 \mathrm{~L})$ was given to the chickens in the fourth chicken house during the first seven days of life.

Ten birds per chicken house were dissected at days 11, 20 and 33 of life. The animals were killed without interventions before being dissected. Anesthesia and the killing of the birds were carried out in accordance with Annex 2 (to Section 2, paragraph 2) of the Regulations on the Welfare of Animals Used for Experiments or for Other Scientific Purposes (TierSchVersV). Anesthesia was performed by head stroke. After bleeding, cecal 
contents were removed under sterile conditions, placed in reaction vessels, immediately frozen and stored at $-80^{\circ} \mathrm{C}$ until simultaneous analysis.

\section{2. $16 S$ rRNA Gene and Statistical Analyses}

Microbiota analyses were performed as already described by Hankel et al. [23]. Samples were purified (Kit: BS 365, BioBasic Inc., Markham, ON, Canada) before the hypervariable region $\mathrm{V} 4$ of the $16 \mathrm{~S}$ rRNA gene was amplified in accordance with previously described protocols [56] using primer F515/R806. Amplicons were sequenced on the Illumina MiSeq platform (PE250). The Usearch8.1 software package (http:/ /www.drive5.com/usearch/ accessed on 22 May 2018) was used to assemble, quality control and cluster obtained reads; -fastq_mergepairs -with fastq_maxdiffs 30 was used to merge the reads. Chimeric sequences were identified and removed with the help of cluster_otus (-otu_radius_pct 3) and the Uchime command included in the Usearch8.1 workflow. Quality filtering was set up with the fastq_filter (-fastq_maxee 1); minimum read length, 200 bp before reads were clustered into 97\% ID operational taxonomic units (OTUs). The UPARSE algorithm [57] was used to determine the OTU clusters and representative sequences. Silva database v128 [58] and the RDP Classifier [59] was used for taxonomic assignment with a bootstrap confidence cutoff of $70 \%$. Data visualization and statistical analyses of microbiota were performed with R (version 4.0.3, www.r-project.org accessed on 15 January 2021) and the R-package "phyloseq" (version 1.32.0, https://joey711.github.io/phyloseq/ accessed on 15 January 2021) [60]. OTUs that were not present in at least one sample were pruned. Additionally, OTUs with an abundance $<0.02 \%$ were filtered and finally, samples with fewer than 999 total reads were removed. Reads assigned to Cyanobacteria, Chloroplast and Mitochondria were filtered.

Samples were normalized (transformed to relative abundance) prior to ordination. Factors contributing to the differences in microbial composition of the samples were identified with Permutational multivariate analysis of variance (PERMANOVA) on Bray-Curtis distances. Ordination was performed using the Bray-Curtis dissimilarity-based principal coordinate analysis (PCoA). Pairwise comparisons for Bray-Curtis distances of bacterial communities associated with treatments were tested using Analysis of similarities (ANOSIM) within the R-package "vegan" (version 2.5.6, https:/ /rdrr.io/cran/vegan/ accessed on 8 August 2021) [61]. The species richness estimators Observed Species, Chao 1 and Shannon index were used to measure sample diversity. A Kruskal-Wallis rank sum test was performed to compare the sample diversity indices of all groups, while additionally each of the groups were compared pairwise to the reference group (lincospectin-treated chickens) using the Wilcoxon rank sum test with the $p$-value adjustment method: holm. Relative abundances of the 50 most abundant OTUs belonging to bacterial phyla were shown as bar charts for days 11, 20, and 33. R-package DESeq2 (version 1.33.4, http:// bioconductor.org/packages/release/bioc/vignettes/DESeq2/inst/doc/DESeq2.html accessed on 8 August 2021) [62] was used to find taxa with significantly different abundance using the Wald test for significance testing, while $p$-values were adjusted by the Benjamini and Hochberg $(\mathrm{BH})$ method to control the false discovery rate (FDR) of 5\%. Relative abundances of the dominant families within the phyla Bacteroidetes and Firmicutes, which at the same time also showed significant differences between the lincospectin-treated and non-treated chickens, and families that were significantly enriched in lincospectin-treated chickens compared to all three other groups were shown.

\section{Conclusions}

It can be concluded that the microbiota composition in the present study was influenced lastingly until slaughter age by the treatment of lincospectin only within the first days of life. At day 11, the microbiota composition of chickens treated with B. licheniformis were most similar to chickens treated with lincospectin, immediately followed by chickens treated with esterified butyrins, making these two additives among the tested ones the most interesting for further studies. Nevertheless, it should be underlined that the potential 
of $B$. licheniformis and water soluble esterified butyrins to prevent enterococcal spondylitis has still to be proven. Future studies with regard to pathogen enterococcal strains might focus on the parameters of enterococcal colonization, intestinal bacteria and intestinal barrier in the early life stages of chickens. A better understanding of these interactions could help in the development of strategies to prevent or treat this disease.

Supplementary Materials: The following are available online at https:/ /www.mdpi.com/article/ $10.3390 /$ pathogens10081068/s1, Table S1. $p$-values ${ }^{1}$ of pairwise comparisons of species richness estimators in cecal samples of chickens, Table S2. Families with significant different abundance between lincospectin and the administered feed additives at day 11, Figure S1. Volcano plot showing families with significant different abundance (FDR-adjusted $p$-values $<0.01$ ) and absolute $\log 2$ fold change $>2$ between chickens treated with Lincospectin and: (a) B. licheniformis, (b) esterified butyrins or (c) palm oil, at day 11 .

Author Contributions: Conceptualization, M.T. and C.V.; methodology, J.H., B.B., M.T., E.G., T.S., D.R., A.A. and C.V.; validation, J.H., B.B., M.T., E.G., T.S., D.R., A.A. and C.V.; formal analysis, J.H. and E.G.; investigation, B.B., D.R., A.A. and C.V.; resources, M.T. and C.V.; data curation, J.H., and B.B.; writing—original draft preparation, J.H.; writing—review and editing, J.H. and C.V.; visualization, J.H.; supervision, C.V.; project administration, C.V.; funding acquisition, C.V. All authors have read and agreed to the published version of the manuscript.

Funding: This research received no external funding. This publication was supported by Deutsche Forschungsgemeinschaft and University of Veterinary Medicine Hannover, Foundation within the funding programme Open Access Publishing.

Institutional Review Board Statement: The animals were killed in accordance with § 4, paragraph 3 of the Animal Protection Act exclusively to use their organs or tissues for scientific purposes. The experiment was approved by the Animal Welfare Officer of the University of Veterinary Medicine Hannover, Germany (reference: TiHo-T-2018-14).

Informed Consent Statement: Not applicable.

Data Availability Statement: The data presented in this study are openly available in NCBI BioProject repository (https:/ / www.ncbi.nlm.nih.gov / bioproject/PRJNA741093 accessed on 24 June 2021), reference number (PRJNA741093).

Acknowledgments: We would like to thank Frances Sherwood-Brock for proofreading the manuscript to ensure correct English.

Conflicts of Interest: The authors declare no conflict of interest.

\section{References}

1. Dolka, B.; Chrobak-Chmiel, D.; Czopowicz, M.; Szeleszczuk, P. Characterization of pathogenic Enterococcus cecorum from different poultry groups: Broiler chickens, layers, turkeys, and waterfowl. PLoS ONE 2017, 12, e0185199. [CrossRef] [PubMed]

2. Herdt, P.d.; Defoort, P.; Steelant, J.V.; Swam, H.; Tanghe, L.; Goethem, S.v.; Vanrobaeys, M. Enterococcus cecorum osteomyelitis and arthritis in broiler chickens. Vlaams Diergeneeskd. Tijdschr. 2009, 78, 44-48.

3. Stalker, M.J.; Brash, M.L.; Weisz, A.; Ouckama, R.M.; Slavic, D. Arthritis and osteomyelitis associated with Enterococcus cecorum infection in broiler and broiler breeder chickens in Ontario, Canada. J. Vet. Diagn. Investig. 2010, 22, 643-645. [CrossRef] [PubMed]

4. Jung, A.; Chen, L.R.; Suyemoto, M.M.; Barnes, H.J.; Borst, L.B. A review of Enterococcus cecorum infection in poultry. Avian Dis. 2018, 62, 261-271. [CrossRef] [PubMed]

5. Martin, L.T.; Martin, M.P.; Barnes, H.J. Experimental reproduction of enterococcal spondylitis in male broiler breeder chickens. Avian Dis. 2011, 55, 273-278. [CrossRef]

6. Borst, L.; Suyemoto, M.; Sarsour, A.; Harris, M.; Martin, M.; Strickland, J.; Oviedo, E.; Barnes, H. Pathogenesis of enterococcal spondylitis caused by Enterococcus cecorum in broiler chickens. Vet. Pathol. 2017, 54, 61-73. [CrossRef]

7. Kense, M.; Landman, W.J. Enterococcus cecorum infections in broiler breeders and their offspring: Molecular epidemiology. Avian Pathol. 2011, 40, 603-612. [CrossRef]

8. Armour, N.K.; Collett, S.R.; Williams, S.M. Enterococcus cecorum-related arthritis and osteomyelitis in broilers and broiler breeders. Poult. Inf. Prof. 2011, 117, 1-7.

9. Robbins, K.M.; Suyemoto, M.M.; Lyman, R.L.; Martin, M.P.; Barnes, H.J.; Borst, L.B. An Outbreak and Source Investigation of Enterococcal Spondylitis in Broilers Caused by Enterococcus cecorum. Avian Dis. 2012, 56, 768-773. [CrossRef] 
10. Walker, G.K.; Suyemoto, M.M.; Gall, S.; Chen, L.; Thakur, S.; Borst, L.B. The role of Enterococcus faecalis during co-infection with avian pathogenic Escherichia coli in avian colibacillosis. Avian Pathol. 2020, 49, 589-599. [CrossRef]

11. Borst, L.; McLamb, K.; Suyemoto, M.; Chen, L.; Levy, M.; Sarsour, A.; Cordova, H.; Barnes, H.; Oviedo-Rondón, E. Coinfection with Eimeria spp. decreases bacteremia and spinal lesions caused by pathogenic Enterococcus cecorum. Anim. Feed Sci. Technol. 2019, 250, 59-68. [CrossRef]

12. Sharma, P.; Gupta, S.K.; Barrett, J.B.; Hiott, L.M.; Woodley, T.A.; Kariyawasam, S.; Frye, J.G.; Jackson, C.R. Comparison of Antimicrobial Resistance and Pan-Genome of Clinical and Non-Clinical Enterococcus cecorum from Poultry Using Whole-Genome Sequencing. Foods 2020, 9, 686. [CrossRef] [PubMed]

13. Stępień-Pyśniak, D.; Marek, A.; Banach, T.; Adaszek, Ł.; Pyzik, E.; Wilczyński, J.; Winiarczyk, S. Prevalence and antibiotic resistance of Enterococcus strains isolated from poultry. Acta Vet. Hung. 2016, 64, 148-163. [CrossRef] [PubMed]

14. Allen, H.K.; Levine, U.Y.; Looft, T.; Bandrick, M.; Casey, T.A. Treatment, promotion, commotion: Antibiotic alternatives in food-producing animals. Trends Microbiol. 2013, 21, 114-119. [CrossRef] [PubMed]

15. Hofacre, C.L.; Mathis, G.F.; Lumpkins, B.S.; Sygall, R.; Vaessen, S.; Hofacre, C.S.; Smith, J.A.; Clanton, E. Efficacy of Butyric and Valeric Acid Esters in a Necrotic Enteritis Challenge Model. Avian Dis. 2020, 64, 407-414. [CrossRef]

16. Timbermont, L.; Lanckriet, A.; Dewulf, J.; Nollet, N.; Schwarzer, K.; Haesebrouck, F.; Ducatelle, R.; Van Immerseel, F. Control of Clostridium perfringens-induced necrotic enteritis in broilers by target-released butyric acid, fatty acids and essential oils. Avian Pathol. 2010, 39, 117-121. [CrossRef]

17. Clausen, M.R.; Mortensen, P.B. Kinetic studies on the metabolism of short-chain fatty acids and glucose by isolated rat colonocytes. Gastroenterology 1994, 106, 423-432. [CrossRef]

18. McIntyre, A.; Gibson, P.; Young, G. Butyrate production from dietary fibre and protection against large bowel cancer in a rat model. Gut 1993, 34, 386-391. [CrossRef]

19. Dalmasso, G.; Nguyen, H.T.T.; Yan, Y.; Charrier-Hisamuddin, L.; Sitaraman, S.V.; Merlin, D. Butyrate transcriptionally enhances peptide transporter PepT1 expression and activity. PLoS ONE 2008, 3, e2476. [CrossRef] [PubMed]

20. Inan, M.S.; Rasoulpour, R.J.; Yin, L.; Hubbard, A.K.; Rosenberg, D.W.; Giardina, C. The luminal short-chain fatty acid butyrate modulates NF-кB activity in a human colonic epithelial cell line. Gastroenterology 2000, 118, 724-734. [CrossRef]

21. Peng, L.; He, Z.; Chen, W.; Holzman, I.R.; Lin, J. Effects of Butyrate on Intestinal Barrier Function in a Caco-2 Cell Monolayer Model of Intestinal Barrier. Pediatr. Res. 2007, 61, 37. [CrossRef]

22. Mariadason, J.M.; Barkla, D.H.; Gibson, P.R. Effect of short-chain fatty acids on paracellular permeability in Caco-2 intestinal epithelium model. Am. J. Physiol. Gastrointest. Liver Physiol. 1997, 272, G705-G712. [CrossRef]

23. Hankel, J.; Jung, K.; Kuder, H.; Keller, B.; Keller, C.; Galvez, E.; Strowig, T.; Visscher, C. Caecal Microbiota of Experimentally Camyplobacter jejuni-Infected Chickens at Different Ages. Front. Microbiol. 2019, 10, 2303. [CrossRef]

24. Onrust, L.; Baeyen, S.; Haesebrouck, F.; Ducatelle, R.; Van Immerseel, F. Effect of in feed administration of different butyrate formulations on Salmonella Enteritidis colonization and cecal microbiota in broilers. Vet. Res. 2020, 51, 1-15. [CrossRef]

25. Fernández, S.M.; Cretenet, M.; Bernardeau, M. In vitro inhibition of avian pathogenic Enterococcus cecorum isolates by probiotic Bacillus strains. Poult. Sci. 2019, 98, 2338-2346. [CrossRef] [PubMed]

26. Xu, S.; Lin, Y.; Zeng, D.; Zhou, M.; Zeng, Y.; Wang, H.; Zhou, Y.; Zhu, H.; Pan, K.; Jing, B. Bacillus licheniformis normalize the ileum microbiota of chickens infected with necrotic enteritis. Sci. Rep. 2018, 8, 1744. [CrossRef] [PubMed]

27. Hovorková, P.; Laloučková, K.; Skřivanová, E. Determination of In Vitro Antibacterial Activity of Plant Oils Containing MediumChain Fatty Acids against Gram-Positive Pathogenic and Gut Commensal Bacteria. Czech. J. Anim. Sci. 2018, 63, 119-125. [CrossRef]

28. Aviagen. Ross 308 Broiler: Performance Objectives. Available online: https://www.google.com/url?sa=t\&rct=j\&q= \&esrc=s\&source=web\&cd=\&ved=2ahUKEwi-1dHHjpzwAhVJgf0HHZzyD3QQFjAAegQIBhAD\&url=https \%3A\%2F\%2Fwww . winmixsoft.com\%2Ffiles\%2Finfo\%2FRoss-308-Broiler-PO-2014-EN.pdf\&usg=AOvVaw2FfKFdmg8PopdgG2t-Dl51 (accessed on 26 April 2021).

29. Ward, T.L.; Weber, B.P.; Mendoza, K.M.; Danzeisen, J.L.; Llop, K.; Lang, K.; Clayton, J.B.; Grace, E.; Brannon, J.; Radovic, I. Antibiotics and Host-Tailored Probiotics Similarly Modulate Effects on the Developing Avian Microbiome, Mycobiome, and Host Gene Expression. MBio 2019, 10, e02171-19. [CrossRef]

30. Zwirzitz, B.; Pinior, B.; Metzler-Zebeli, B.; Handler, M.; Gense, K.; Knecht, C.; Ladinig, A.; Dzieciol, M.; Wetzels, S.U.; Wagner, M. Microbiota of the Gut-Lymph Node Axis: Depletion of Mucosa-Associated Segmented Filamentous Bacteria and Enrichment of Methanobrevibacter by Colistin Sulfate and Linco-Spectin in Pigs. Front. Microbiol. 2019, 10, 599. [CrossRef] [PubMed]

31. Ruiz, V.E.; Battaglia, T.; Kurtz, Z.D.; Bijnens, L.; Ou, A.; Engstrand, I.; Zheng, X.; Iizumi, T.; Mullins, B.J.; Müller, C.L. A single early-in-life macrolide course has lasting effects on murine microbial network topology and immunity. Nat. Commun. 2017, 8, 1-14. [CrossRef] [PubMed]

32. Tonks, A.A. Exploring the Effects of Management Strategies on the Gut Microbiome and Metabolome of Growing Broiler Chickens: An Integrated Metagenomic and Metabolomic Approach. Ph.D. Thesis, University of Reading, Reading, UK, 2018.

33. Pereira, R.; Bortoluzzi, C.; Durrer, A.; Fagundes, N.S.; Pedroso, A.A.; Rafael, J.M.; Perim, J.E.d.L.; Zavarize, K.C.; Napty, G.S.; Andreote, F.D. Performance and intestinal microbiota of chickens receiving probiotic in the feed and submitted to antibiotic therapy. J. Anim. Physiol. Anim. Nutr. 2019, 103, 72-86. [CrossRef] 
34. Fjalstad, J.W.; Esaiassen, E.; Juvet, L.K.; van den Anker, J.N.; Klingenberg, C. Antibiotic therapy in neonates and impact on gut microbiota and antibiotic resistance development: A systematic review. J. Antimicrob. Chemother. 2018, 73, 569-580. [CrossRef]

35. Turnbaugh, P.J.; Hamady, M.; Yatsunenko, T.; Cantarel, B.L.; Duncan, A.; Ley, R.E.; Sogin, M.L.; Jones, W.J.; Roe, B.A.; Affourtit, J.P. A core gut microbiome in obese and lean twins. Nature 2009, 457, 480-484. [CrossRef] [PubMed]

36. Richards, P.; Wigley, P.; Fothergill, J.; Bernardeau, M. Development of the Caecal Microbiota in Three Broiler Breeds. Front. Vet. Sci. 2019, 6, 201. [CrossRef] [PubMed]

37. Oakley, B.B.; Buhr, R.J.; Ritz, C.W.; Kiepper, B.H.; Berrang, M.E.; Seal, B.S.; Cox, N.A. Successional changes in the chicken cecal microbiome during 42 days of growth are independent of organic acid feed additives. BMC Vet. Res. 2014, 10, 282. [CrossRef] [PubMed]

38. Kubasova, T.; Kollarcikova, M.; Crhanova, M.; Karasova, D.; Cejkova, D.; Sebkova, A.; Matiasovicova, J.; Faldynova, M.; Pokorna, A.; Cizek, A. Contact with adult hen affects development of caecal microbiota in newly hatched chicks. PLoS ONE 2019, 14, e0212446. [CrossRef] [PubMed]

39. Han, Z.; Li, L.; Willer, T.; Baumgärtner, W.; Rautenschlein, S. Adhesion and invasion of Campylobacter jejuni in chickens with a modified gut microbiota due to antibiotic treatment. Vet. Microbiol. 2020, 240, 108504. [CrossRef]

40. Jacobs-Reitsma, W.; Van de Giessen, A.; Bolder, N.; Mulder, R. Epidemiology of Campylobacter spp. at two Dutch broiler farms. Epidemiol. Infect. 1995, 114, 413-421. [CrossRef]

41. Stern, N.J.; Cox, N.A.; Musgrove, M.T.; Park, C. Incidence and Levels of Campylobacter in Broilers After Exposure to an Inoculated Seeder Bird. J. Appl. Poult. Res. 2001, 10, 315-318. [CrossRef]

42. Rychlik, I. Composition and Function of Chicken Gut Microbiota. Animals 2020, 10, 103. [CrossRef]

43. Ocejo, M.; Oporto, B.; Hurtado, A. 16S rRNA amplicon sequencing characterization of caecal microbiome composition of broilers and free-range slow-growing chickens throughout their productive lifespan. Sci. Rep. 2019, 9, 2506. [CrossRef]

44. Videnska, P.; Sedlar, K.; Lukac, M.; Faldynova, M.; Gerzova, L.; Cejkova, D.; Sisak, F.; Rychlik, I. Succession and replacement of bacterial populations in the caecum of egg laying hens over their whole life. PLoS ONE 2014, 9, e115142. [CrossRef]

45. Stackebrandt, E. The family Lachnospiraceae. In The Prokaryotes, 4th ed.; Rosenberg, E., DeLong, E.F., Lory, S., Stackebrandt, E., Thompson, F., Eds.; Springer: Berlin/Heidelberg, Germany, 2014; pp. 197-201.

46. Vacca, M.; Celano, G.; Calabrese, F.M.; Portincasa, P.; Gobbetti, M.; De Angelis, M. The Controversial Role of Human Gut Lachnospiraceae. Microorganisms 2020, 8, 573. [CrossRef]

47. Biddle, A.; Stewart, L.; Blanchard, J.; Leschine, S. Untangling the Genetic Basis of Fibrolytic Specialization by Lachnospiraceae and Ruminococcaceae in Diverse Gut Communities. Diversity 2013, 5, 627-640. [CrossRef]

48. Guilloteau, P.; Martin, L.; Eeckhaut, V.; Ducatelle, R.; Zabielski, R.; Van Immerseel, F. From the gut to the peripheral tissues: The multiple effects of butyrate. Nutr. Res. Rev. 2010, 23, 366-384. [CrossRef]

49. Chen, L.; Zhong, R.; Zhang, L.; Zhang, H. The Chronic Effect of Transgenic Maize Line with mCry1Ac or maroACC gene on Ileal Microbiota Using a Hen Model. Microorganisms 2019, 7, 92. [CrossRef]

50. Feng, Y.; Wang, L.; Khan, A.; Zhao, R.; Wei, S.; Jing, X. Fermented wheat bran by xylanase-producing Bacillus cereus boosts the intestinal microflora of broiler chickens. Poult. Sci. 2020, 99, 263-271. [CrossRef]

51. Patterson, J.; Burkholder, K. Application of prebiotics and probiotics in poultry production. Poult. Sci. 2003, 82, 627-631. [CrossRef] [PubMed]

52. Abd El, A.E.-M.E.; El-Wardany, I.; Abu-Taleb, A.M.; Wakwak, M.M.; Ebeid, T.A.; Saleh, A.A. Assessment of in ovo administration of Bifidobacterium bifidum and Bifidobacterium longum on performance, ileal histomorphometry, blood hematological, and biochemical parameters of broilers. Probiotics. Antimicro. Prot. 2020, 12, 439-450. [CrossRef]

53. Chen, Y.-C.; Yu, Y.-H. Bacillus licheniformis-fermented products improve growth performance and the fecal microbiota community in broilers. Poult. Sci. 2020, 99, 1432-1443. [CrossRef] [PubMed]

54. Trela, J.; Kierończyk, B.; Hautekiet, V.; Józefiak, D. Combination of Bacillus licheniformis and salinomycin: Effect on the growth performance and git microbial populations of broiler chickens. Animals 2020, 10, 889. [CrossRef] [PubMed]

55. Zaghari, M.; Sarani, P.; Hajati, H. Comparison of two probiotic preparations on growth performance, intestinal microbiota, nutrient digestibility and cytokine gene expression in broiler chickens. J. Appl. Anim. Res. 2020, 48, 166-175. [CrossRef]

56. Caporaso, J.G.; Lauber, C.L.; Walters, W.A.; Berg-Lyons, D.; Lozupone, C.A.; Turnbaugh, P.J.; Fierer, N.; Knight, R. Global patterns of 16S rRNA diversity at a depth of millions of sequences per sample. Proc. Nat. Acad. Sci. USA 2011, 108, 4516-4522. [CrossRef] [PubMed]

57. Edgar, R.C. UPARSE: Highly accurate OTU sequences from microbial amplicon reads. Nat. Methods 2013, 10, 996. [CrossRef] [PubMed]

58. Quast, C.; Pruesse, E.; Yilmaz, P.; Gerken, J.; Schweer, T.; Yarza, P.; Peplies, J.; Glockner, F. The SILVA ribosomal RNA gene database project: Improved data processing and web-based tools. Nucleic Acids Res. 2013, 41, D590-D596. [CrossRef]

59. Wang, Q.; Garrity, G.M.; Tiedje, J.M.; Cole, J.R. Naive Bayesian classifier for rapid assignment of rRNA sequences into the new bacterial taxonomy. Appl. Environ. Microbiol. 2007, 73, 5261-5267. [CrossRef] [PubMed]

60. McMurdie, P.J.; Holmes, S. phyloseq: An R package for reproducible interactive analysis and graphics of microbiome census data. PLoS ONE 2013, 8, e61217. [CrossRef] 
61. Oksanen, J.; Blanchet, F.; Friendly, M.; Kindt, R.; Legendre, P.; McGlinn, D.; Minchin, P.; O’Hara, R.; Simpson, G.; Solymos, P. Vegan: Community Ecology Package, R Package Version 2.5-6; 2019. Available online: https://CRAN.R-project.org/package=vegan (accessed on 8 August 2021).

62. Love, M.I.; Huber, W.; Anders, S. Moderated estimation of fold change and dispersion for RNA-seq data with DESeq2. Genome Biol. 2014, 15, 550. [CrossRef] 